\title{
Marijuana Effects on Sensitivity to Reinforcement in Humans
}

\author{
Scott D. Lane, Ph.D., and Don R. Cherek, Ph.D.
}

Under controlled laboratory conditions, eight adult subjects smoked placebo and three different potencies of marijuana cigarettes ranging in $\Delta^{9}$ THC content. Immediately following smoking, subjects were exposed to a laboratory task that provided concurrently available response options. One option systematically decreased in reinforcement frequency throughout the session, and thus required a reallocation of behavior to the non-decreasing option to maximize monetary earnings. After smoking the two highest doses ( $1.77 \%$ and $\left.3.58 \% \Delta^{9} \mathrm{THC}\right)$ subjects earned fewer reinforcers and allocated a higher proportion of responding to the decreasing option, compared with placebo and the lowest dose. The difference in reinforcers earned could not be accounted for by a change in response rates. Quantitative and graphical analyses revealed that the higher doses produced considerable periods of time spent on the decreasing option despite earning few reinforcers. The data are discussed with regard to marijuana effects on dopamine/cannabinoid systems and adaptive behavior change.

[Neuropsychopharmacology 26:520-529, 2002] (C) 2002 American College of Neuropsychopharmacology. Published by Elsevier Science Inc.
KEY WORDS: Marijuana; Humans; Reinforcement; Laboratory task; Learning; Adaptive behavior change

Administration of marijuana has consistently been shown to disrupt behavioral and CNS functions, including acquisition and choice discrimination learning, short-term memory, and time perception (Adams and Barratt 1976; Chait and Pierri 1992; Darley et al. 1973; Heishman et al. 1990; Kelly et al. 1993; Schulze et al. 1989; Vachon et al. 1974). These acute effects-which are evident in rats, monkeys, and humans-are presumably related to the disruptive action of $\Delta^{9} \mathrm{THC}$ in the brain. With regard to learning processes, some labo-

From the Department of Psychiatry and Behavioral Sciences, University of Texas Health Science Center - Houston.

Address correspondence to: Scott D. Lane, Ph.D., Dept of Psychiatry and Behavioral Sciences, UTSHC-Houston, 1300 Moursund St. Houston, TX 77030, Tel.: (713) 500-2620, Fax: (713) 500-2618, E-mail: scott.d.lane@uth.tmc.edu

Received May 14, 2001; revised August 17, 2001; accepted September 24, 2001.

Online publication: 9/26/01 at www.acnp.org/citations/Npp 092601179 . ratory tasks like repeated acquisition and match to sample are shaped and maintained by reinforcement, e.g., food, money, or positive feedback (Kelly et al. 1993; Schulze et al. 1989). However, studies of acute marijuana effects on reinforcement-based learning have focused on the acquisition of response patterns rather than sensitivity to reinforcer frequency or magnitude, and less is known about the interaction between acute marijuana intoxication and behavioral sensitivity to reinforcement frequency.

Two questions logically precede an inquiry into the effects of marijuana on sensitivity to reinforcement. Why would one expect any relationship? Why would such a relationship be important? At the behavioral level, acute administration of $\Delta^{9} \mathrm{THC}$ has repeatedly been shown to impair performance on learning tasks shaped and maintained by reinforcement (Brady and Balster 1980, Kelly et al. 1993; Kamien et al. 1994; Schulze et al. 1989). Furthermore, on simulated tasks in which subjects could work to earn money, acute marijuana smoking decreased rates of working (and earnings)_an effect presumably mediated by changes in 
reinforcement or "motivational" processes (Foltin et al. 1989; Haney et al. 1997, Kagel et al. 1980; Miles et al. 1974; Pihl and Sigal 1978).

While the present study was not intended to address specific mechanisms, activity at the biochemical level provides a plausible rationale for disruptive effects of $\Delta^{9}$ THC on reinforcement processes. Marijuana impacts both dopamine (DA) and cannabinoid (CB) receptor systems. Administration of $\Delta^{9}$ THC modifies DA release in the "reward pathway", or mesolimbic DA system, projecting from the ventral tegmental area to the nucleus accumbens and into prefrontal cortex (Chen et al. 1990, Gardner and Lowinson 1991, Tanda et al. 1997). Since this pathway is a primary site involved in reinforcement processes (Fibiger and Phillips 1988; Koob and Swerdlow 1988; Wise and Rompre 1989), it follows that marijuana may have acute effects on behavioral sensitivity to reinforcement (see also Loeber and Yurgelun-Todd 1999). $\Delta^{9}$ THC also directly modifies $\mathrm{CB}$ receptor function, including sites in the amygdala and hippocampus (Herkenham 1995; Heyser et al. 1993). CB receptors may regulate the adaptive learning processes via facilitation of long-term potentiation (Kreitzer and Regehr 2001; Wilson and Nicoll 2001). The flooding of $\mathrm{CB}$ receptors by acute doses of marijuana can disrupt this process (Barinaga 2001; Hampson and Deadwyler 2000), and may subsequently affect reinforcement-based learning. Disruption of reinforcement processes in the form of impaired sensitivity to changes in reinforcement contingencies has significant implications. Sensitivity to consequences, and specifically to reinforcer frequency, is a critical process in adaptive behavior (Newland et al. 1994; Staddon 1998). Individuals able to efficiently modify behavior patterns in accord with changes in environmental contingencies are conferred a decided advantage over potential competitors (Skinner 1984). For humans, this may have implications in areas such as social interaction, and academic or workplace performance.

The present study was intended to determine the effects of acute marijuana smoking on behavioral sensitivity to changes in reinforcer frequency in humans. We used a concurrent schedule design with schedule values that changed within-session. The advantages of concurrent schedule preparations in measuring reinforcer sensitivity have been discussed at length previously (e.g., Herrnstein 1997; Mazur 1991), including measurement of drug effects on reinforcement sensitivity (Bacotti 1980; Heyman 1983). This design allowed for assessment of relative response proportions across placebo and three active doses of marijuana that varied in $\Delta^{9}$ THC content. If marijuana alters reinforcement processes at the behavioral and biochemical levels, we hypothesized that acute doses would disrupt sensitivity to frequency of reinforcement as measured by relative response proportions across changing conditions.

\section{METHOD}

\section{Subjects}

Eight adults (six males, 2 females, age 19-40) participated after signing an informed consent agreement approved by the local IRB. All subjects reported occasional marijuana use defined as 2 to 10 times per month, as well as past use of at least one other drug, including alcohol, cocaine, opiates, and benzodiazepines. Subjects were recruited via local newspaper advertisements for "behavioral research". None of the subjects had previously participated in research studies. Based on information obtained during initial telephone interviews, potential subjects were brought to the laboratory for more extensive interviews covering physical and mental health status, and drug and alcohol use history. Exclusion criteria included: (1) current or past medical problems (e.g., traumatic head injury, high blood pressure, asthma); (2) current use of any medications; (3) current ongoing drug use; and (4) current or past history of an Axis I disorder other than substance dependence, as defined by the Structured Clinical Interview for the DSM-IV (SCID-I, version 2.0, First et al. 1996).

Regarding the exclusionary criterion of current ongoing drug use: while subjects reporting marijuana use 2-10 times per month were recruited into the study, prior to any testing they were required to provide a clean urine sample. Following each active dose administered in the study, a clean urine sample was required before the next active dose was administered. This typically took 3-4 days following an active dose, and active doses were separated by at least seven days. After beginning the study, subjects' participation was discontinued following two consecutive drug-positive urine samples (confirmed by daily urinalysis) or breath alcohol samples. This requirement was used to rule out a potential interaction of acute marijuana and residual effects from extra-experimental drug use, including marijuana. Urine drug screen analysis was carried out using enzyme multiple immunoassay (EMIT d.a.u ${ }^{\circledR}$, SYLVA Corp). Temperature monitoring and creatinine determinations were performed to detect attempts to alter urine samples.

\section{Apparatus}

During experimental sessions, subjects worked alone in a $1.2 \times 1.8 \mathrm{~m}$, sound-attenuating test chamber equipped with a $36.5 \mathrm{~cm}\left(14^{\prime \prime}\right)$ VGA color monitor, and a $10.0 \times$ $43.0 \times 25.0 \mathrm{~cm}$ response panel with three Microswitch buttons labeled A, B, and C. Experimental events and data collection were handled by a remote MS Windows-based PC and a Med Associates model 750 interface card, using custom software written in MicroSoft Visual Basic. 


\section{Subject Payment and Schedule}

Subjects were paid daily for performance during experimental sessions and attendance, and were given a completion bonus at the end of the experiment. The testing protocol lasted approximately four weeks, with subjects participating either two or three days per week, dictated by their schedules. During the first two days of the study, subjects were given an examination by a physician and provided initial exposure to the laboratory task (no marijuana doses were administered). The physical examination served to ensure subjects were free of any medical conditions that would preclude participation. Initial exposure to the task served to stabilize response rates prior to initiating dose administration.

Each day of the study, subjects arrived at approximately 8:00 A.M. Breath and urine samples were collected at $\sim 8: 15$ A.M. Subjects participated in either four or five experimental sessions, lasting either 25 or 50 min, depending on the experimental condition (details below). The first testing session began at 8:30 A.M., prior to administration of the dose for that day. Following dose administration at 9:30, subjects completed either three (10:00 A.M., 1:00 P.M. and 2:00 P.M.) or four (10:00 A.M., 11:30 A.M., 1:00 P.M. and 2:00 P.M.) more test sessions, depending on the test schedule in effect (see below). Between sessions, subjects stayed in a waiting room with magazines, books, and a TV. Lunch was provided at 12:00 P.M. Subjects were not allowed to eat any other food or smoke cigarettes between 8:00 A.M. and 2:30 P.M. Compliance with non-smoking instructions was verified by expired CO samples taken in the morning and afternoon. Five of eight subjects reported smoking daily with a mean of 11.80 cigarettes per day (range 2-20). Formal data regarding nicotine withdrawal were not collected but no subjects reported symptoms during the study.

\section{Behavioral Testing Procedures}

During most sessions of the study, subjects responded under a multiple random interval schedule of reinforcement, hereafter designated Multiple RI. In a random interval reinforcement schedule, intervals of time are selected from a distribution with a specified average and range and are recycled randomly throughout the session. Any response that is made after the selected time interval has elapsed produces a reinforcing outcome (e.g., the addition of money). The procedure generates relatively constant response rates, since each interval in the distribution has an equal probability of occurring. The present schedule used values ranging between 1 and $10 \mathrm{~s}$, and averaging $5 \mathrm{~s}$. Each response made after the selected time interval (1 to $10 \mathrm{~s}$ ) had elapsed added $\$ 0.03$ to the subjects earnings, and the next interval was selected. At the beginning of the session a monetary counter located near the top center of the monitor screen read " $\$ 0.00$ " and a letter was located near the middle of screen, either $C$ on the left or $A$ on the right. Pressing the button that corresponded to the letter shown on the screen produced monetary reinforcers (\$0.03) according to the RI $5 \mathrm{~s}$ schedule. Each session was $24 \mathrm{~min}$, and the active response option shown on the screen ( $\mathrm{C}$ or $\mathrm{A})$ alternated every $6 \mathrm{~min}$. The option shown on screen at the beginning of the session was alternated between sessions.

On five occasions, during the second session only and immediately following dose administration (see details below), subjects worked on a concurrent random interval schedule of reinforcement, hereafter designated Concurrent RI. Figure 1 provides a depiction of the Concurrent RI procedure. The random interval schedule functioned identically to that described above, with a specified average and range of intervals. However, instead of a single response option, both options (C and A) were available at any time during the session. To switch between options, subjects were required to press the middle (B) button five times. Only the letter corresponding to the selected option was shown on the screen. This process is shown in Figure 1. A separate
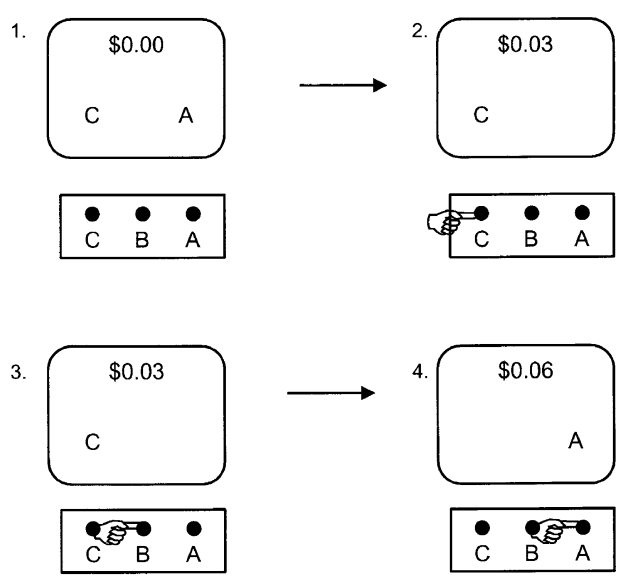

Figure 1. Depiction of the Concurrent Random Interval procedure that immediately followed marijuana administration (Session 2). The numbered screens and associated text describe the sequence of events as they occurred from the beginning of the session: (1) Start of session, both response options available; (2) Subject selects button $C$ and begins responding, the letter $\mathrm{A}$ is removed from the screen, money is added to the counter at a rate of $\$ 0.03$ every $5 \mathrm{~s}$; (3) Subject responds 5 times on button B; (4) Subject begins responding on button $A$. On the first response, the letter $C$ is removed from the screen, the letter A appears. Subject begins earning money responding on button $\mathrm{A}$. The subject is free to switch back and forth between buttons $\mathrm{A}$ and $\mathrm{C}$ as long as the changeover requirement ( 5 presses on button $\mathrm{B}$ ) occurred first. The letters do not change and money cannot be earned without a proper changeover. As session progresses, the buttons produce reinforcers $(\$ 0.03)$ at systematically changing rates (see text for details). The subject is required to allocating responding appropriately to maximize earnings. 
Random Interval schedule was associated with each response option. During the first $10 \mathrm{~min}$ of the session, the two RI schedule values were identical, averaging RI $5 \mathrm{~s}$ each with a range of 1 to $10 \mathrm{~s}$. Thereafter, every 10 min one of the concurrently available response options changed in schedule value and, as a result decreased in reinforcement frequency. Response proportions during minutes $0-10$ determined the decreasing option; whichever option was preferred ( $>0.50$ response allocation) became the option that decreased in reinforcer frequency. The decreasing option changed according to the following schedule values: $\min 11-20=$ average $15 \mathrm{~s}$ (interval range 3-30); min 21-30 = average $20 \mathrm{~s}$ (range $4-40$ ); $\min 31-40=$ average $25 \mathrm{~s}$ (range 5-50); $\min 41-50=$ average $30 \mathrm{~s}$ (range 6-60). The selected range of interval values was purposely restricted so that discriminations could be made, and appropriate behavioral transition observed, within a single 50-min session.

\section{Instructions}

Prior to the first Multiple RI session on Day 1, subjects received instructions about the stimulus display (letters and monetary counter shown on screen), and the function of responding on the letter shown on the monitor screen. They were informed that they would be required to switch back and forth between response options and that the counter would keep a running total of their earnings.

Prior to the first Concurrent RI session, immediately following the placebo administration, subjects received instructions that both the letters $C$ and A would appear on the screen at the same time, and that they could respond on either button at any time during the session, as long as they pressed the B button five times prior to switching options. In subsequent Concurrent RI sessions, subjects were reminded of these instructions immediately prior to the start of the session.

\section{Marijuana Cigarettes and Administration}

Marijuana cigarettes supplied by NIDA were used and ranged across four doses: placebo cigarettes containing $0.0001 \% \mathrm{w} / \mathrm{w} \Delta^{9} \mathrm{THC}$, and the following active doses: half of $1.77 \% \Delta^{9}$ THC (1/2 placebo and $1 / 2$ active cigarette); $1.77 \% \Delta^{9}$ THC (both halves active); and 3.58\% $\Delta^{9}$ THC (both halves active). Cigarettes were stored at $-20^{\circ} \mathrm{C}$ and cut in half and humidified before smoking. Cigarettes were smoked in a ventilated enclosed chamber.

Subjects smoked the two halves of one marijuana cigarette immediately prior to the beginning of the second experimental session of the day ( $\sim 9: 30$ A.M.). Smoking was cued by a series of colored lights, associated via instructions with the following sequence: $2 \mathrm{~s}$ blue light $=$ get ready; $3 \mathrm{~s}$ green light $=$ inhale, $10 \mathrm{~s}$ red light $=$ hold your breath, all lights off $=$ exhale. Lights remained off for $30 \mathrm{~s}$ and the sequence repeated continuously until both halves of the cigarette were smoked. The ventilated smoking chamber exhausted air to the outside of the building.

Rather than hold constant the number of inhalations, all cigarette halves were smoked down to a pencil mark $1 / 4$ inch from the end. In procedures that administer inhaled drugs, the following relevant parameters have been documented: drug concentration (e.g., THC content), inhalation volume, number of inhalations, breathhold duration, and inter-inhalation interval (Azorlosa et al. 1992, 1995; Zacny et al. 1987). The procedure used here controlled for these parameters except number of inhalations, which was free to vary across subjects and doses, but was always recorded (see data analyses). Because of individual differences in lung capacity and smoking history, inhalation volume typically varies across subjects. Here, volume was controlled as closely as possible by cueing the length of the inhalation with a (green) discriminative stimulus light. More precise control over inhalation volume has been accomplished through the use of custom-designed electromechani$\mathrm{cal} /$ physiological equipment and software (Azorlosa et al. 1995). However, even when these more elaborate procedures have been used, heart rate and subjective effects data (the most well validated measures of acute marijuana intoxication) were not markedly different than those obtained with the administration procedures used here.

Control over number of inhalations versus amount of cigarette smoked involves a trade off. If subjects make a different number of inhalations across doses, it could result in differences in volume of marijuana smoked. However, subjects often compensate for THC content by titrating inhalations at higher doses and require more inhalations to smoke an equivalent amount of the higher THC doses compared with placebo (Heishman et al. 1988; Kelly et al. 1993). Thus, holding the number of inhalations constant across doses can result in differences in smoking volume (see Azorlosa et al. 1992). Number of inhalations per cigarette half was recorded and compared across all conditions. Most importantly, the current paced, cued smoking procedure has been widely used and produces reliable physiological and subjective effects data indicative of acute marijuana intoxication (Chait 1989; Cherek et al. 1993; Haney et al. 1997).

\section{Dosing Sequence}

Initially, subjects worked only under the Multiple RI schedule and smoked only placebo cigarettes until response rates stabilized and urine cannabinoid levels were zero. Dosing then ensued in the following sequence of conditions: placebo - active - active - active placebo. On each of those five days, Session 1 (8:30) was 
a Multiple RI condition, Session 2 (immediately after smoking) was a 50 min Concurrent RI session, and Sessions 3 and 4 (1:00 P.M., 2:30 P.M.) were Multiple RI conditions. In between each of the five dosing days that involved the Concurrent RI schedule, subjects returned to placebo cigarettes and only Multiple RI sessions until responding was stable and urine cannabinoid levels returned to zero. This typically entailed either one or two experimental days. The THC content of the active doses was counterbalanced such that each one of the six possible orders was completed by the first six subjects. The final two subjects were quasi-balanced such that neither dose occurred in the same position (i.e., half-1.77, 1.77, 3.58 and $1.77,3.58$, half-1.77).

The atypical dosing sequence (placebos before and after the three active doses) and the limited exposure to the Concurrent RI session (only during Session 2 on the five main dose days) was constructed after pilot data revealed that multiple exposures to the Concurrent RI schedule under conditions of no drug engendered longterm discrimination of the change in schedule values (i.e., the increasing RI value on the initially preferred option). After repeated exposures, the changing Concurrent RI task lost sensitivity because, rather than having to discriminate changes in reinforcer frequency, subjects simply chose an initial option then later switched to the other option and remained there until the session was over (not optimal, but a successful strategy once the changing schedule contingency was recognized). Thus, the dosing sequence was chosen to limit baseline/placebo exposures to the Concurrent RI schedule prior to encountering active doses. The second placebo dose allowed for assessment of the discrimination via comparison of the first and second exposures under placebo.

\section{Cardiovascular and Self-Report Measures}

Immediately prior to and following marijuana smoking the subject's heart rate, systolic and diastolic blood pressure were measured using an automatic oscillometric digital blood pressure and pulse monitor (Marshall Electronics, Inc., Lincolnshire, IL). Immediately after the post-smoking cardiovascular measures subjects completed a rating form. They were asked to estimate the subjective effects of the marijuana cigarette on a five-point scale (anchored by " 0 - not at all" and " $4-$ extremely") by rating the following statements: "I feel an effect of the marijuana smoke," "My heart is pounding faster than normal," "I feel dizzy, lightheaded," and "I feel a typical marijuana high."

\section{Data Analyses}

The dependent measures related to marijuana administration were analyzed by repeated measures ANOVA.
For number of inhalations, a two-way repeated measures ANOVA was conducted with factors of cigarette half $\left(1^{\text {st }}, 2^{\text {nd }}\right)$ and dose $\left(1^{\text {st }}\right.$ placebo, half of $1.77 \%, 1.77 \%$, $3.58 \%, 2^{\text {nd }}$ placebo). For the cardiovascular and subjective effects data, one-way repeated measures ANOVAs were conducted with repeated measures on dose (placebo, half of $1.77 \%, 1.77 \%, 3.58 \%$ ). Tukey tests of all pairwise comparisons were used for post-hoc analyses.

The behavioral data were analyzed across three comprehensive dependent measures and one more detailed dependent measure. All behavioral data were taken from the five sessions in which subjects were exposed to the Concurrent RI schedule (Session \#2 on the five primary dose days). The three molar-level dependent measures were total number of reinforcers earned, overall proportion of responses allocated to the response option that decreased in reinforcement frequency, and the total number of responses across both options. The latter variable (total responses) was included because of the possibility that overall response rates might have been differentially changed as a function of dose. Substantial decreases in total responses could have affected the number of reinforcers earned because available reinforcers would be missed. In other words, a subject could obtain a significantly different number of reinforcers on two different days either by allocating a relatively large proportion of responses to the decreasing option or by simply not responding rapidly enough to maximize available reinforcer rates on either option.

To assess the relationship between response allocation and reinforcers earned on a more detailed level, the data were partitioned into 10-min segments and graphed with response proportions on the $\mathrm{Y}$ axis as a function of reinforcers earned on the $X$ axis, then fitted to the power function $y=a x^{b}$, where the exponents $a$ and $b$ refer to the 0 intercept and the rate of increase (slope), respectively. The shape of these functions was quantified using the index of curvature, a measure devised to describe non-linear response patterns on time-based reinforcement schedules (see Fry et al. 1960). Any negatively accelerating curve produces a negative curvature index; any positively accelerating curve produces a positive index (Fry et al. 1960). In the present data, a more negatively accelerating curve indicates a high proportion of responses on the decreasing option despite few reinforcers being earned. The curvature indexes derived for each of the four doses were compared by $t$-tests, using residual variance from the non-linear regression analyses (see Zarr 1984 for details on the use of the $t$-test involving regression analysis parameters).

To assess the possibility of order effects confounding the results (e.g., the "switch and stay" strategy following permanent discrimination of the changing schedule contingency noted above), a number addi- 
tional statistical analysis were performed on the behavioral data. For the three comprehensive dependent measures - total reinforcers obtained, overall response proportions, and total responses - the data were analyzed in two ways. First, the first and second placebo conditions were compared via $t$-tests. Second, the active dose data were partitioned by order of administration, rather than dose, and compared by one-way repeated measures ANOVA. Significant outcomes on any of these comparisons would be indicative of order effects.

\section{RESULTS}

\section{Marijuana Administration}

A two-way repeated measures ANOVA on the number of inhalations revealed a significant main effect of dose, $\mathrm{F}_{4,28}=5.94, p<.002$. Tukey post-hoc comparisons showed differences between the following conditions: $1.77 \%$ vs. the first placebo, $p<.02 ; 1.77 \%$ vs. the second placebo, $p<.05 ; 1.77 \%$ vs. half- $1.77 \%, p<.05$; and $3.58 \%$ vs. the first placebo, $p<.03$. The next highest difference in the means was between $3.58 \%$ and the second placebo, but the difference did not reach significance, $p<.09$. There was not a significant main effect of cigarette half, $\mathrm{F}_{1,7}=0.40$, and there was not a significant interaction of dose by cigarette half, $\mathrm{F}_{4,28}=0.59$.

\section{Cardiovascular and Subjective Effects}

Table 1 shows means and standard errors for the cardiovascular and self-report measures, and outcomes from 1-way ANOVAs with repeated measures across dose. There was a significant effect of marijuana dose on heart rate change from pre- to post-smoking, $\mathrm{F}_{3,21}=$ $14.26, p<.001$. Tukey post-hoc analyses revealed significant differences between the placebo and 3.58\% $(p<$ $.001), 1.77 \%(p<.001)$, and half-1.77\% $(p<.05)$ doses. There was not a significant effect of dose on either systolic blood pressure, $\mathrm{F}_{3,21}=1.05$, or diastolic blood pres- sure, $\mathrm{F}_{3,21}=0.817$. For the subjective effects data, there was a significant effect of dose on the rating "I feel an effect of marijuana smoke", $\mathrm{F}_{3,21}=12.03, p<.001$. Significant differences on post-hoc tests were observed between placebo and all three active doses: $3.58 \%, p<$ $.001 ; 1.77 \%, p<.002$; and half- $1.77 \%, p<.002$. There was a significant effect of dose on the rating "My heart is pounding faster than normal", $\mathrm{F}_{3,21}=7.92, p<.002$. Significant differences on post-hoc tests were observed between placebo and 3.58\%, $p<.002$, and $1.77 \%, p<$ .006. There was a significant effect of dose on the rating "I feel dizzy, light-headed", $\mathrm{F}_{3,21}=5.15, p<.009$. Significant differences on post-hoc tests were observed only between placebo and $3.58 \%, p<.005$. There was a significant effect of dose on the rating "I feel a typical marijuana high", $\mathrm{F}_{3,21}=11.09, p<.001$. Significant differences on post-hoc tests were observed between placebo and all three active doses: $3.58 \%, p<.001 ; 1.77 \%, p<$ .003 ; half- $1.77 \%, p<.005$.

\section{Behavioral Data}

Figure 2 shows the total number of reinforcers earned (left $\mathrm{Y}$ axis) and the total number of responses made (right $\mathrm{Y}$ axis) across placebo and the three active doses. The horizontal line near the bottom of the graph shows the minimum number responses (1250) that would be required to earn all available reinforcers on the RI 5 schedule (assuming a consistent response rate across the session). Clearly, subjects were responding well in excess of the minimum requirement. As can be seen in the graph, reinforcers earned decreased as a function of dose: $3.58 \%=323.38( \pm 65.55), 1.77 \%=331.13$ $( \pm 71.86)$, half- $1.77 \%=419.25( \pm 110.12)$, placebo $=$ 439.65 ( \pm 85.32). This difference was significantly different, $\mathrm{F}_{3,21}=5.47, p<.006$. Tukey post-hoc tests revealed significant differences between placebo and $3.58 \%, p<.01$, and between placebo and $1.77 \%, p<.02$. The total number of responses decreased at the higher doses: $3.58 \%=3286.88( \pm 1160.60) ; 1.77 \%=2789.63$

Table 1. Mean ( \pm S.E.M.) Cardiovascular and Subjective Effects Following Marijuana Administration. Values for Heart Rate, Systolic and Diastolic Blood Pressure Represent Change Scores (Post-Minus Pre-Smoking). The Rightmost Column Shows Statistical Outcomes from Comparisons Across Doses with 1-Way with Repeated Measures ANOVA

\begin{tabular}{|c|c|c|c|c|c|}
\hline Dependent Measure value & Placebo & half- $1.77 \%$ & $1.77 \%$ & $3.58 \%$ & $\mathrm{~F}_{3,21}, p$ \\
\hline$\Delta$ Heart Rate & $+6.6(1.40)$ & $+17.63(3.27)$ & $+34.00(6.49)$ & $+29.86(6.00)$ & $14.26,<.001$ \\
\hline$\Delta$ Systolic BP & $+5.67(2.67)$ & $-7.38(6.26)$ & $-1.13(4.74)$ & $-6.25(5.05)$ & $1.05, \mathrm{~ns}$ \\
\hline$\Delta$ Diastolic BP & +3.13 (1.94) & $+4.25(6.30)$ & $-4.75(5.47)$ & $-0.75(3.42)$ & $0.82, \mathrm{~ns}$ \\
\hline $\begin{array}{l}\text { "I feel an effect of } \\
\text { marijuana smoke" }\end{array}$ & $1.40(0.27)$ & $2.25(0.41)$ & $2.25(0.45)$ & $2.63(0.42)$ & $12.03,<.001$ \\
\hline $\begin{array}{l}\text { "My heart is pounding } \\
\text { faster than normal" }\end{array}$ & $0.53(0.17)$ & $1.13(0.40)$ & $1.63(0.32)$ & $1.88(0.35)$ & $7.92,<.002$ \\
\hline $\begin{array}{l}\text { "I feel dizzy, light-headed" } \\
\text { "I feel a typical }\end{array}$ & $0.53(0.19)$ & $0.63(0.18)$ & $0.75(0.31)$ & $1.38(0.46)$ & $5.15,<.007$ \\
\hline marijuana high" & $1.13(0.34)$ & $2.13(0.44)$ & $2.25(0.53)$ & $2.75(0.45)$ & $11.09,<.001$ \\
\hline
\end{tabular}




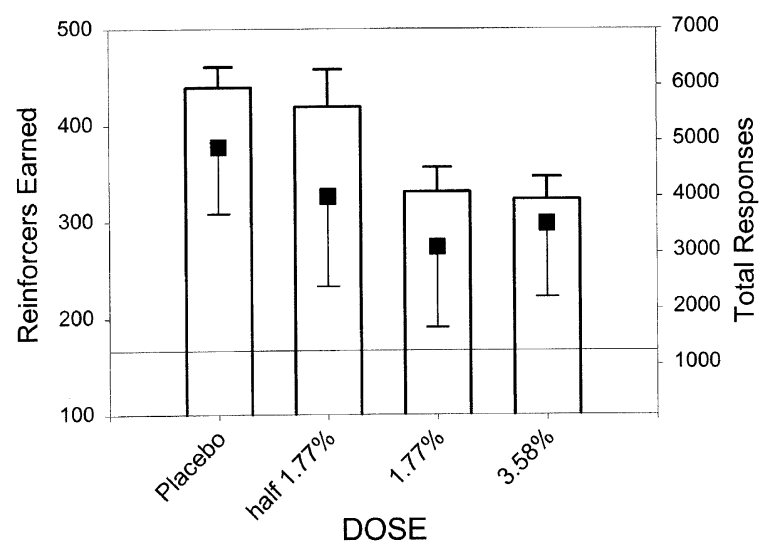

Figure 2. Average number of reinforcers earned (bars, left $\mathrm{Y}$ axis) and total responses made (squares, right $\mathrm{Y}$ axis) at each of the four doses used in the experiment. The data represent behavior during the Concurrent Random Interval (Session 2) that was presented immediately following marijuana administration. The horizontal line near the bottom is plotted on the right $\mathrm{Y}$ axis, and represents an approximate value (1250) that would be needed to obtain all scheduled reinforcers when the schedule value was at RI $5 \mathrm{~s}$, assuming a consistent response rate across the session (see text for details). Error bars represent 1 S.E.M.

$( \pm 1280.30) ;$ half- $1.77 \%=3511.88( \pm 1474.00)$, placebo $=$ 4434.91 ( \pm 1092.06). This difference, however, was not significantly different, $\mathrm{F}_{3,21}=2.72$.

Subjects earned fewer reinforcers as a function of dose, but responded at a rate sufficient to obtain all reinforcers available even on the schedule component with the highest reinforcement rate (RI 5). Thus, the differences in reinforcers earned can be attributed to differential response allocation on the two options, and cannot be attributed to sedation or decreased response rates. Consequently, the proportion of responses allocated to the decreasing response option, collapsed across each of the 10-min blocks, increased as a function of dose: $3.58 \%=0.51( \pm 0.06), 1.77 \%=0.50( \pm 0.06)$, half-1.77\% $=0.21( \pm 0.05)$, placebo $=0.23( \pm .03)$. This difference was statistically significant, $\mathrm{F}_{3,117}=14.24, p<$ .001 . Tukey post-hoc tests revealed significant difference between placebo and 3.58\%, $p<.001$; half- $1.77 \%$ and $3.58 \%, p<.002$; placebo and $1.77 \%, p<.001$; and half $-1.77 \%$ and $1.77 \%, p<.003$.

As described in the methods, order effects could have influenced the data. To assess this possibility, each of the preceding dependent measures was analyzed by comparing the first and second placebo administrations, and by comparing the three active doses partitioned by administration order rather than $\Delta^{9}$ THC content. The total number of reinforcers earned was not significantly different between the first and second placebo administration. Mean values were 426.88 ( \pm 29.45$)$ and $452.38( \pm 32.20), t(7)=-0.99$. Total number of re- inforcers earned was also not significantly different across the active dose administration days. Mean values were $383.75( \pm 42.20)$ for day $1,352.63( \pm 19.05)$ for day 2 , and $341.50( \pm 33.69)$ for day $3, F_{2,14}=0.36$. The total number of responses made was not significantly different between the first and second placebo administration. Mean values were 4609.56 ( \pm 1767.06$)$ and 4260.25 $( \pm 1407.07), \mathrm{t}(7)=0.25$. Total number of responses was also not significantly different across the active dose days. Mean values were 3242.50 ( \pm 1341.66) for day 1, 3236.38 $( \pm 1339.84)$ for day 2 , and 3109.50 ( \pm 1265.32$)$ for day 3 , $\mathrm{F}_{2,14}=0.05$. The proportion of responses allocated to the decreasing option was not significantly different between the first and second placebo administration. Mean values were $0.21( \pm 0.04)$ and $0.26( \pm 0.05), \mathrm{t}(39)=-1.48$. The proportion of responses allocated was also not significantly different across the active dose days. Mean values were $0.35( \pm 0.06)$ for day $1,0.46( \pm 0.06)$ for day 2 , and $0.41( \pm 0.06)$ for day $3, F_{2,78}=0.89$.

Figure 3 shows a more detailed analysis of the response proportion data, plotted as proportion of re-
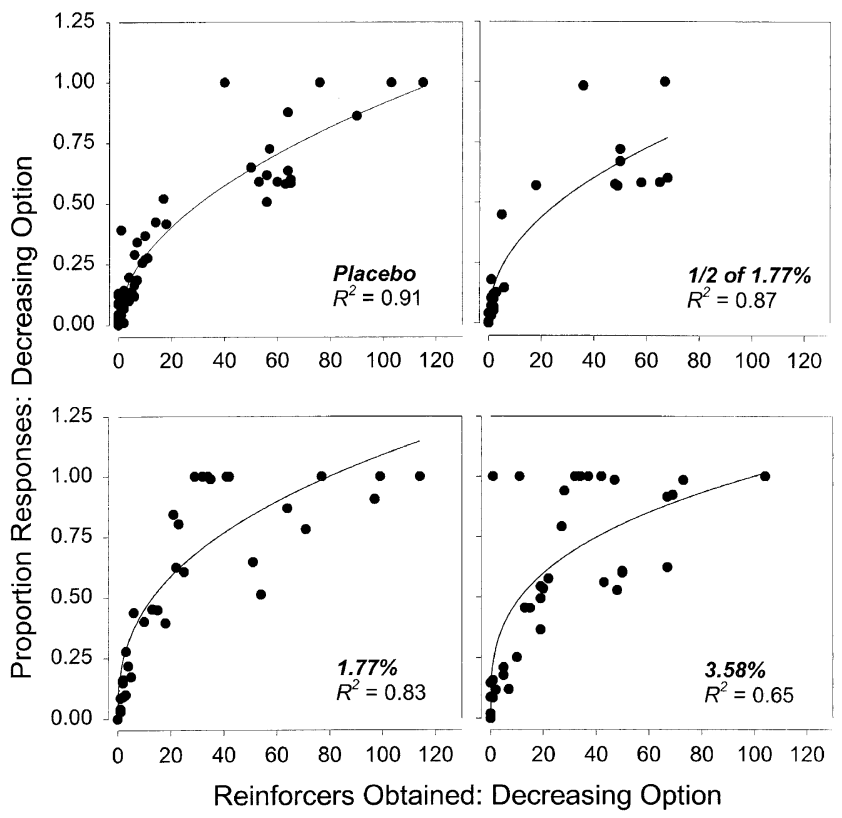

Figure 3. The proportion of responses ( $\mathrm{Y}$ axis) made on the option decreasing in reinforcer frequency every $10 \mathrm{~min}$ in the last $40 \mathrm{~min}$ of a $50 \mathrm{~min}$ session (Session 2), plotted as a function of reinforcers obtained on that decreasing option ( $X$ axis). Each data point represents one 10 min segment for one subject. Response proportions for all eight subjects are plotted in each panel. Each panel shows one of the four doses used in the experiment. The functions (solid lines) were derived by fitting the data to the non-linear equation $y=a x^{b}$ : a negatively accelerating function that accounted for $91 \%$ of the variance under placebo conditions, demonstrating that response allocations were sensitive to reinforcer frequency (upper left panel, see text for further details). Variance $\left(R^{2}\right)$ is shown in the lower right of each panel. 
sponses on the decreasing option as a function of number of reinforcers earned. Each data point represents a 10-min block during the decreasing Concurrent RI session; all subjects are plotted together for each dose. The data were fitted to the non-linear equation $y=a x^{b}$, a negatively accelerating function that accounted for the relationship between response allocation and reinforcers earned quite well under placebo conditions $\left(R^{2}=\right.$ 0.91) - suggesting that individual response allocations were sensitive to the decreasing reinforcer frequency. This relationship decreased as a function of dose; $\mathrm{R}^{2}$ values for half- $1.77 \%, 1.77 \%$, and $3.58 \%$ were $0.87,0.83$ and 0.65 , respectively. This trend can be observed in the number of data points in the upper left quadrant of each graph. Data points in this area indicate high response allocations on the decreasing option despite few reinforcers earned. This relationship was quantified by the index of curvature, in which increasingly negative values indicate a more steeply decelerating negative trend - caused by data in the upper left sector of the graph (see methods for details). The index of curvature values became more negative as a function of dose: placebo $=-0.52$, half- $1.77 \%=-0.56,1.77 \%=-0.63$, and $3.58 \%=-0.69 . t$-tests revealed these differences in the index of curvature values to be statistically significant for placebo versus $3.58 \%, t(16)=-4.31, p<.001$; placebo versus $1.77 \%, t(16)=-3.51, p<.01$; and half$1.77 \%$ versus $3.58 \%, t(16)=-2.73, p<.02$. There were no significant differences between placebo and half$1.77 \%, t(16)=-1.65 ;$ half- $1.77 \%$ and $1.77 \%, t(16)=$ -1.92 ; or $1.77 \%$ and $3.58 \%, t(16)=-1.32$.

\section{DISCUSSION}

Subjects were administered placebo and three active marijuana doses ranging in $\Delta^{9}$ THC content. Immediately following smoking, subjects were exposed to a laboratory task that provided concurrent response options. One option systematically decreased in reinforcement frequency throughout the session, requiring the reallocation of behavior to the non-decreasing option to maximize reinforcer intake (i.e., monetary earnings). At the two highest doses $\left(1.77 \%\right.$ and $3.58 \% \Delta^{9}$ THC) subjects earned fewer reinforcers and allocated a higher proportion of responding to the decreasing option than after smoking either placebo or the low dose. The difference in reinforcers earned could not be accounted for by an overall decline in response rates, and after smoking high doses subjects spent considerable periods of time on the decreasing option despite earning few reinforcers. Below we discuss these outcomes, their implications for adaptive behavior in humans, and limitations of the current experiment.

Subjects required significantly more inhalations to smoke the $1.77 \%$ and $3.58 \%$ cigarettes compared with placebo. The chemical extraction process of THC from placebo cigarettes renders them dryer than non-extracted cigarettes. Thus, they tend to burn faster and may require fewer inhalations. Furthermore, despite attempts to control inhalation volume via discriminative cues, titration of smoke volume is regularly observed in studies administering smoked marijuana to humans (Heishman et al. 1988; Kelly et al. 1993). As $\Delta^{9}$ THC content increases, subjects typically reduce the strength of inhalations presumably to control the rapid onset of psychoactive effects that follow smoking high THC-content marijuana (Heishman et al. 1989). Despite this outcome, measures of heart rate and subjective effects reliably increased as a function of THC content, indicating that the administration procedures had the intended dose-related effect. Specifically, requiring subjects to smoke each cigarette half down to the $1 / 4$ inch mark engendered equivalent smoke intake each dose despite titration. Requiring subjects to take the same number of inhalations at each dose may well have produced uncontrolled variance in smoke intake as a result of titration.

The behavioral data indicate that marijuana acutely disrupted sensitivity to changes in reinforcement frequency. The two highest doses $(1.77 \%$ and $3.58 \%)$ produced markedly different response patterns under the concurrent RI schedule with decreasing reinforcement frequency, highlighted by periods of time in which a high proportion of responses were allocated to the decreasing response option despite the fact that few if any reinforcers were being earned. In other words, subjects continued to respond on an option that provided monetary rewards less and less often over time.

Subjects earned significantly less money at the $1.77 \%$ and $3.58 \%$ doses. Notably, these doses also produced a decrease in the total number of responses emitted, which could have been responsible for the decrease in earnings. The suppressing effect of $\Delta^{9} \mathrm{THC}$ on response rates has been observed in several previous studies (Cherek et al. 1993; Schulze et al. 1989; Winsauer et al. 1999). Importantly, average rates did not drop below the minimum rate that would be required to maximize reinforcer intake (even at the RI 5 schedule). Importantly, response rates were not significantly different across doses and do not account for the observed outcomes on response adaptation to the RI schedule changes.

The observed effects were not uniformly dosedependent. It is not uncommon in marijuana studies for changes in behavior not to be linearly related to dose. Most commonly, the outcomes divide into placebo/low and moderate/high dose effects. The variables controlling this high versus low dose effect are not well understood, but could be related to the idiosyncratic changes in inhalation patterns that are observed as THC-content increases (Heishman et al. 1989).

The biobehavioral mechanisms that may have been involved in the present experiment can only be dis- 
cussed at a general level, as the design of this study did not allow for direct inference. Adaptive behavior on this task required two courses of action: (1) discrimination of a change reinforcement frequency, and (2) a subsequent shift of behavior away from the decreasing response option. Frequency is a measure of count over time, and accurate discrimination requires some consistent perception of elapsed time. Marijuana has been reliably demonstrated to distort time perception (Chait and Pierri 1992; Darley et al. 1973), which could in turn distort discrimination of the frequency at which an event occurs. Another possibility is that $\Delta^{9}$ THC-induced changes in neurotransmission interfere with the discriminative and reinforcing properties acquired by stimuli during association-based learning. For example, recent data suggest that endogenous cannabinoids in the hippocampus may help facilitate long-term potentiation (Kreitzer and Regehr 2001; Wilson and Nicoll 2001), and overwhelming of CB receptors by acute marijuana may disrupt this process (Hampson and Deadwyler 2000). These events could alter the properties of relevant stimuli during learning tasks that require adaptive behavior change. The stimulation of DA transmission in the reward pathway resulting from acute $\Delta^{9}$ THC administration (Gardner and Lowinson 1991, Tanda et al. 1997) raises the possibility that the reinforcing efficacy of stimuli may be altered by transient dysregulation of normal DA function. With regard to the present data, we find this an intriguing and plausible possibility which merits further, but more direct, investigation.

Replication of the current finding and confirmatory studies with broader external validity will be necessary to validate the present observation that marijuana acutely disrupts sensitivity to changes in reinforcement frequency, and therefore adaptability to environmental changes. If this relationship is more thoroughly substantiated, it has meaningful applied implications. Sensitivity to reinforcement contingencies is a critical component in adaptive behavior (Skinner 1984; Staddon 1998), and in human social settings this bears on effective interpersonal communication (e.g., discrimination of verbal and non-verbal cues, see also Higgins and Stitzer 1986), and academic and workplace performance. Perhaps equally as important is the possibility that such marijuana-related impairments extend beyond the time periods of acute intoxication to lasting residual effects. Residual impairments on learning and performance tasks have been observed in both humans (Block and Ghoneim 1993; Pope and Yurgelun-Todd 1996) and monkeys (Paule et al. 1992).

\section{ACKNOWLEDGMENTS}

This study was supported by grant DA 12968 from the National Institutes of Health. We thank Joel L. Steinberg, M.D., for helpful scientific advice, and Sheila White and Jennifer Sharon for technical assistance.

\section{REFERENCES}

Adams PM, Barratt ES (1976): The effects of marijuana extract on two-choice discrimination learning in the squirrel monkey. Phys Psychology 4:155-158

Azorlosa JL, Greenwald MK, Stitzer ML (1995): Marijuana smoking: effects of varying puff volume and breathhold duration. J Pharmacol Exp Ther 272:560-569

Azorlosa JL, Heishman SJ, Stitzer ML, Mahaffey JM (1992): Marijuana smoking: effect of varying delta 9-tetrahydrocannabinol content and number of puffs. J Pharmacol Exp Ther 261:114-122

Bacotti AV (1980): Effects of cocaine and morphine on concurrent schedule-controlled performances. J Pharmacol Exp Ther 212:280-286

Barinaga M (2001): How cannabinoids work in the brain. Science 291:2530-2531

Block RI, Ghoneim MM (1993): Effects of chronic marijuana use on human cognition. Psychopharmacology 110:219-228

Brady KT, Balster RL (1980): The effects of delta 9-tetrahydrocannabinol alone and in combination with cannabidiol on fixed-interval performance in rhesus monkeys. Psychopharmacology 72:21-26

Chait LD (1989): Delta-9-tetrahydrocannabinol content and human marijuana self- administration. Psychopharmacology 98:51-55

Chait LD, Pierri J (1992): Effects of smoked marijuana on human performance. In Murphy L, Bartke A (eds), Marijuana/Cannabinoids: Neurobiology and Neurophysiology. Boca Raton, FL, CRC Press, pp 387-423

Chen J, Paredes W, Lowinson JH, Gardner EL (1990): $\Delta^{9}$-Tetrahydrocannabinol enhances presynaptic dopamine efflux in medial prefrontal cortex. Eur J Pharmacol 190:259-262

Cherek DR, Roache JD, Egli M, Davis C, Spiga R, Cowan K (1993): Acute effects of marijuana smoking on aggressive, escape and point-maintained responding of male drug-users. Psychopharmacology 111:163-168

Darley CF, Tinklenberg JR, Hollister LE, Atkinson RC (1973): Marijuana and retrieval from short-term memory. Psychopharmacologia 29:231-238

Fibiger H, Phillips A (1988): Mesocorticolimbic dopamine systems and reward. In Kalivas PW, Nemeroff CB (eds), The Mesocorticolimbic Dopamine System. New York, Ann N Y Acad Sci, pp 206-215

First MB, Spitzer RL, Gibbon M, Williams JBW (1996): Structured clinical interview for DSM-IV Axis I Disorders: Non patient edition (SCID-NP), 2.0 ed. New York, NY State Psychiatric Institute

Foltin RW, Fischman MW, Brady JV, Kelly TH, Bernstein DJ, Nellis MJ (1989): Motivational effects of smoked marijuana: behavioral contingencies and high-probability recreational activities. Pharmacol Biochem Behav 34:871-877

Fry W, Kelleher RT, Cook L (1960): A mathematical index of performance on fixed-interval shcedules of reinforcement. J Exp Anal Behav 3:193-199 
Gardner EL, Lowinson JH (1991): Marijuana's interaction with brain reward systems: update 1991. Pharmacol Biochem Behav 40:571-580

Hampson RE, Deadwyler SA (2000): Cannabinoids reveal the necessity of hippocampal neural encoding for shortterm memory in rats. J Neuroscience 20:8932-8942

Haney M, Comer SD, Ward AS, Foltin RW, Fischman MW (1997): Factors influencing marijuana self-administration by humans. Behav Pharmacol 8:101-112

Heishman SJ, Huestis MA, Henningfield JE, Cone EJ (1990): Acute and residual effects of marijuana: profiles of plasma THC levels, physiological, subjective, and performance measures. Pharmacol Biochem Behav 37:561-565

Heishman SJ, Stitzer ML, Bigelow GE (1988): Alcohol and marijuana: comparative dose effect profiles in humans. Pharmacol Biochem Behav 31:649-655

Heishman SJ, Stitzer ML, Yingling JE (1989): Effects of tetrahydrocannabinol content on marijuana smoking behavior, subjective reports, and performance. Pharmacol Biochem Behav 34:173-179

Herkenham M (1995): Localization of cannabinoid receptors in brain and periphery. In Pertwee R (ed), Cannabinoid receptors. Academic Press, London, pp 145-166

Herrnstein RJ (1997): The Matching Law. The Harvard University Press, Cambridge MA

Heyman GM (1983): A parametric evaluation of the hedonic and motoric effects of drugs: pimozide and amphetamine. J Exp Anal Behav 40:113-122

Heyser CJ, Hampson RE, Deadwyler SA (1993): Effects of delta-9-tetrahydrocannabinol on delayed match to sample performance in rats: alterations in short-term memory associated with changes in task specific firing of hippocampal cells. J Pharmacol Exp Ther 264: 294-307

Higgins ST, Stitzer ML (1986): Acute marijuana effects on social conversation. Psychopharmacology 90:160-162

Kagel JH, Battalio RC, Miles CG (1980): Marihuana and work performance: results from an experiment. J Hum Resources 15:373-395

Kamien JB, Bickel WK, Higgins ST, Hughes JR (1994): The effects of $\Delta^{9}$-tetrahydrocannabinol on repeated acquisition and performance of response sequences and on self-reports in humans. Behav Pharmacol 5:71-78

Kelly TH, Foltin RW, Fischman MW (1993): Effects of smoked marijuana on heart rate, drug ratings, and task performance by humans. Behav Pharmacol 4:167-178

Koob GF, Swerdlow N (1988): The functional output of the mesolimbic dopamine system. In Kalivas PW, Nemeroff CB (eds), The Mesolimbic Dopamine System. New York, Ann N Y Acad Sci, pp 216-227

Kreitzer AC, Regehr WG (2001): Retrograde inhibition of presynaptic calcium influx by endogenous cannabinoids at excitatory synapses onto Purkinje cells. Neuron 29:717-727

Loeber RT, Yurgelun-Todd DA (1999): Human neuroimag- ing of acute and chronic marijuana use: Implications for frontocerebellar dysfunction. Hum Psychopharmacol Clin Exp 14:291-304

Mazur JE (1991): Choice. In Iversen IH, Lattal KA (eds), Experimental Analysis of Behavior: Part 1. New York, Elsevier, pp 219-250

Miles CG, Congreve GR, Gibbins RJ, Marshman J, Devenyi P, Hicks RC (1974): An experimental study of the effects of daily cannabis smoking on behaviour patterns. Acta Pharmacol Toxicol 34 (Suppl 1):1-44

Newland MC, Yezhou S, Logdberg B, Berlin M (1994): Prolonged behavioral-effects of in-utero exposure to lead or methyl mercury - reduced sensitivity to changes in reinforcement contingencies during behavioral transitions and in steady-state. Tox App Pharmacol 126:6-15

Paule MG, Allen RR, Bailey JR, Scallet AC, Ali SF, Brown RM, Slikker WJ (1992): Chronic marijuana smoke exposure in the rhesus monkey. II: Effects on progressive ratio and conditioned position responding. J Pharmacol Exp Ther 260:210-222

Pihl RO, Sigal H (1978): Motivation levels and the marihuana high. J Abnorm Psychol 87:280-285

Pope HGJ, Yurgelun-Todd D (1996): The residual cognitive effects of heavy marijuana use in college students. JAMA 275:521-527

Schulze GE, McMillan DE, Bailey JR, Scallet AC, Ali SF, Slikker WJ, Paule MG (1989): Acute effects of marijuana smoke on complex operant behavior in rhesus monkeys. Life Sci 45:465-475

Skinner BF (1984): Selection by consequences. Behav Brain Sci 7:477-510

Staddon JER (1998): Models of action: Mechanisms for Adaptive Behavior. Mahwah, NJ, Lawrence Erlbaum Associates, Inc.

Tanda G, Pontieri FE, Di Chiara G (1997): Cannabinoid and heroin activation of mesolimbic dopamine transmission by a common mu1 opioid receptor mechanism. Science 276:2048-2050

Vachon L, Sulkowski A, Rich E (1974): Marihuana effects on learning, attention and time-estimation. Psychopharmacol 39:1-11

Wilson RI, Nicoll RA (2001): Endogenous cannabinoids mediate retrograde signalling at hippocampal synapses. Nature 410:588-592

Winsauer PJ, Lambert P, Moerschbaecher JM (1999): Cannabinoid ligands and their effects on learning and performance in rhesus monkeys. Behav Pharmacol 10:497-511

Wise RA, Rompre P-P (1989): Brain dopamine and reward. Ann Rev Psychol 40:191-225

Zacny JP, Stitzer ML, Brown FJ, Yingling JE, Griffiths RR (1987): Human cigarette smoking: Effects of puff and inhalation parameters on smoke exposure. J Pharmacol Exp Ther 240:554-564

Zarr JH (1984): Biostatistical Analysis (2nd ed.). Englewood Cliffs, NJ, Prentice-Hall 